\title{
Maternal Expectancy Versus Objective Measures of Child Skill: Evidence for Absence of Positive Bias in Mothers' Expectations of Children with Internalizing Disorders
}

\author{
Catherine M. Udy • Carol Newall • Suzanne Broeren • \\ Jennifer L. Hudson \\ Published online: 29 August 2013 \\ (C) The Author(s) 2013. This article is published with open access at Springerlink.com
}

\begin{abstract}
Parents of anxious children are thought to be more attuned to threat, which might translate into less positive bias in parental report of child coping and ability, unlike parents of non-anxious children. Maternal expectancy bias was examined in a sample of 43 clinically anxious (51\% female), 30 clinically anxious/depressed (50\% female), and 44 nonclinical control children (46\% female), $8-14$ years of age. When compared to an objective observer's ratings of the children, mothers of non-clinical children demonstrated a positive bias (i.e., over-rated their children's performance) for both ratings of expected speech performance in absolute terms and relative to peers. Mothers in the clinical groups did not exhibit this positive expectancy bias. Moreover, mothers of clinical children reported lower expectations in absolute terms and relative to peers than mothers of non-clinical children. The data suggest that mothers of clinical children held accurate expectations for child performance when compared to the gold standard of an objective observer.
\end{abstract}

Keywords Childhood anxiety · Maternal anxiety .

Maternal-report bias $\cdot$ Self-enhancing bias

Positive, self-serving biases have been shown to be a hallmark of mental health well-being (for a review, see Taylor and Brown 1988, 1994). For example, appraising oneself as better

Electronic supplementary material The online version of this article (doi:10.1007/s10802-013-9793-1) contains supplementary material, which is available to authorized users.

C. M. Udy $\cdot$ C. Newall $\cdot$ S. Broeren · J. L. Hudson Centre for Emotional Health, Department of Psychology,

Macquarie University, Sydney, Australia

C. M. Udy · C. Newall · S. Broeren · J. L. Hudson $(\bowtie)$

Department of Psychology, Macquarie University,

Sydney, NSW 2109, Australia

e-mail: jennie.hudson@mq.edu.au than the average person and being overly optimistic about one's performance may serve to enhance one's self-esteem (Taylor and Brown 1988, 1994). Furthermore, this positive illusory bias towards oneself also extends to close others. For instance, parents generally see their own children in a more favorable light than other children and are inclined to overestimate their child's abilities and level of development (e.g., Miller 1986; Miller et al. 1991).

Miller et al. (1991) proposed that parents may be motivated to see their children in a positive light because this enhances the parent's self-esteem. However, when difficulties arise, such as when a child suffers from an internalizing or externalizing disorder, it may be more protective of parental selfesteem if they distanced themselves from the issues by attributing the child's problematic behaviors to stable characteristics within the child. This may alleviate parental sense of responsibility for the child's difficulties. In these cases, parents may be less likely to be influenced by a self-serving, positive bias when predicting their child's performance, and will be more accurate or even more pessimistic reporters of their child's abilities (Gretarsson and Gelfand 1988). There is some evidence to support this idea. For example, results from a study by Gretarsson and Gelfand (1988) indicate that mothers may be less motivated to maintain positive perceptions of their child when the child is more difficult to manage. Furthermore, in samples where children are developmentally delayed, increased accuracy in maternal perception of the child's performance is associated with decreased child IQ and developmental functioning (Malhi et al. 2005). This finding is in line with the suggestion that positive parental bias may wane in situations where children are less able. Taken together, it seems that parents of children who display behavioral or emotional difficulties are less likely to display the positive self-serving biases seen in the general population.

There are several possible explanations for the lack of positive bias among parents of children with behavioural and emotional difficulties. Several researchers have stressed 
the importance of considering informant attributions about the causes of the child's problems, as well as each informant's unique perspective when evaluating a child's difficulties within the context of diagnostic interviews (e.g., Comer and Kendall 2004; Des Los Reyes 2011; Des Los Reyes and Kazdin 2005). For instance, Des Los Reyes and Kazdin (2005) have noted that parents are more likely than children to attribute ongoing problems to the child's disposition rather than the environment or context. Therefore, parents of clinical children may show less of a positive bias because they will expect their child's salient dispositions (i.e., problematic behaviors or emotional problems) to impact negatively on the outcome. Several researchers have also proposed that parents of anxious children may be more attuned to threat and that this threat-bias can be observed when these parents evaluate their child's abilities too (Bar-Haim et al. 2007; Creswell et al. 2005). This threat-bias in parents may manifest in the form of low expectations of the child's coping abilities and is considered to be one possible mechanism by which anxiety may be transmitted from parent to offspring (Rapee 1997). For instance, the mother of an anxious child will often be aware that their child will struggle in anxietyprovoking situations. This will likely increase the parent's perceptions of threat when their child enters a challenging situation, and the parent's concerns about threat may be inadvertently communicated to their children through excessive reassurance and over-involvement. Such parental behaviours may predispose these children to feel less confident and competent, thus increasing the child's anxiety.

To date, only a few studies have addressed the question of how accurately a parent of an anxious child predicts his or her child's skills and coping abilities, and whether these parents have lower expectations of their children than parents of nonanxious children (Cobham et al. 1999; Kendall and Chansky 1991; Kortlander et al. 1997). In a study of 27 clinically anxious and 7 non-anxious control children and their parents, Kendall and Chansky (1991) found that there were no significant differences between parent- and child-reported expectations of child coping in the non-anxious group. In contrast, anxiety-disordered children reported that they expected to cope significantly better than their parents expected them to cope. Kendall and Chansky (1991) suggested that one possible explanation for this discrepancy between parent and child report is that anxious children may be more inclined to "fake good" (p. 179), and that their parents may provide a more accurate prediction of the child's actual coping ability. However, these results do not provide an objective standard to which we can determine the relative accuracy of parent and child reports. It is possible that within the non-clinical group, both parents and children were overly optimistic about the child's coping. While it is clear that the parents of the anxious children had lower expectations of child coping than their children, these predictions could have been either reasonably accurate or overly pessimistic.
Kortlander et al. (1997) included an objective observer in their study on differences in maternal expectations regarding children's coping, level of distress, and level of skill in a stressful speech task between mothers of clinically anxious and non-anxious children. Results showed that mothers of anxious children expected their child to be more upset and less able to cope than did mothers of non-anxious children. They were also less confident of their child's abilities to perform the stressful speech task than mothers of nonanxious children. This was in line with ratings of the objective observer, who rated the anxious children as displaying more anxious behaviors during the task than non-anxious children. Kortlander et al. (1997) therefore suggested that maternal expectations of the child's coping abilities appear to reflect genuinely lower coping abilities of anxious children compared to their non-anxious peers. However, the maternal confidence ratings and the observer's ratings of anxious behaviors were not directly comparable. This meant that while the groups differed both in maternal expectations for speech success and objectively observed anxious behaviors, it is not clear from the data whether mothers of anxious children underestimated their children's ability, or whether mothers of non-anxious controls displayed the illusory positive bias that is typical of non-clinical populations in the literature.

One other aspect of maternal expectations that needs to be addressed is the role of maternal psychopathology in reporting bias. Specifically, higher rates of maternal anxiety and depression in anxious children may contribute to more negative parental predictions and reports. In their review of the evidence for depressive realism, Ackermann and DeRubeis (1991) concluded that depressed and dysphoric individuals were more accurate than non-depressed individuals on selfversus-other judgments, whereas non-depressed individuals tend to exhibit a self-enhancing bias. Furthermore, there is also evidence for a lack of a specific self-serving bias in anxious mothers of anxious children. Cobham et al. (1999) found no significant differences for children's ratings of expected anxiety, skill level or avoidance between clinically anxious, clinical control (i.e., children with an externalizing disorder), and non-clinical control children. However, within the anxious child group, anxious mothers were significantly more likely to predict that their child would choose an avoidant solution than non-anxious mothers of anxious children. Given that all children were alike in their tendency to choose an avoidant solution, this study provides evidence that maternal psychopathology may play a role in the lack of selfserving biases. That is, anxious mothers of anxious children may be more realistic or even pessimistic compared to their non-anxious counterparts, which may manifests itself as a lack of the self-enhancing biases that is usually evident in nonclinical samples. However, the lack of an objective gold standard with which the child's performance can be compared limits the conclusions drawn from the studies reviewed so far. 
The current study examined whether mothers of children with anxiety disorders expected their child to be less skilled and less able to cope in a stressful performance situation than mothers of non-anxious control children. Maternal ratings were compared to objective observer ratings to determine whether patterns in maternal responses reflected accurate assessments of the child's skill and coping ability. The proposition that mothers of anxious children might lack the positive bias seen in non-clinical populations was tested and the possible influence of maternal psychopathology (i.e., anxiety or depression) was also assessed. A co-morbid child anxiety/ depression group was also included as a control group for various reasons. First, anxiety and depression frequently cooccur in child and adolescent samples (e.g., Angold et al. 1999; Kessler et al. 2003; Mathew et al. 2011). Second, depressive symptoms have been shown to reduce selfserving biases (e.g., Ackermann and DeRubeis 1991). Third, parent-child interactions in groups of depressed children have been found to be high in negativity and criticism (Hughes and Gullone 2008; McLeod et al. 2007), which may indicate that parents of depressed children also have less positive expectations of their children. Finally, the inclusion of an anxious/ depressed group also allows us to investigate whether the lack of parental positive bias can be extended more broadly to internalizing disorders rather than specifically to childhood anxiety disorders.

Based on the existing literature, we predicted that mothers of children in the non-anxious group would exhibit a positive bias, rating their own child as more capable than the objective observer on absolute measures of speech performance. Furthermore, these mothers would also expect their child to be better than average on the task relative to their child's peers. In contrast, we hypothesized that mothers of anxious and anxious/depressed children would not demonstrate the same positive bias or would expect their child to perform worse than the average child. We also predicted that maternal expectancies would be lower or more negative in the anxious/depressed group given the evidence that maternal negativity and criticism is associated with depression in childhood and adolescents (Hughes and Gullone 2008; McLeod, et al. 2007). Finally, we predicted that maternal psychopathology, particularly maternal depression, would be associated with more negative maternal expectations of their child's performance.

\section{Method}

\section{Participants}

The total sample comprised 117 mother/child dyads with children aged between 8 and 14 years. Child participants presenting for assessment and treatment at a child and adolescent specialist anxiety clinic were recruited into the group of clinical participants. The research clinic specialises in interventions for anxiety disorders across all age groups, but is most well-known for its research in the treatment of childhood anxiety disorders. There were 43 anxious $(M$ age $=10.70, S D=1.81$, range: $8-14 ; 51 \%$ female) and 30 anxious/depressed children $(M=11.63, S D=1.96$, range: 8 $14 ; 50 \%$ female). A community sample of 44 children was recruited as the control group $(M=11.05, S D=2.07$, range: 8 $14 ; 46 \%$ female) via advertisements to parents in school newsletters and on community notice boards. We also invited parents in a first year psychology subject pool. A majority of participants identified themselves as Australian ( $84.1 \%$ control group, $85.7 \%$ anxious group, and $70 \%$ anxious/ depressed group). Most participating families were middle to high income families, with $74 \%$ of the control group, $46 \%$ of the anxious group, and $62 \%$ of the anxious/depressed group earning over AUS $\$ 80,000$ per annum. No significant differences were found between the families of the three groups on any of demographic variables (age, gender, family income, family make-up, mother's education, and ethnicity; all $p \mathrm{~s}>0.05)$.

The diagnostic status of all participants was established using the parent and child report versions of the Anxiety Disorders Interview Schedule (ADIS-IV-C/P: Silverman and Albano 1996). This instrument has been shown to possess good inter-rater ( $\mathrm{K}=0.80$ to 0.92 for combined diagnoses) and test-retest reliability (Intra-class correlations [ICC] of 0.78 0.96 for symptom scales; Silverman et al. 2001). Participants seeking treatment were interviewed for diagnostic status by postgraduate psychology students and clinical psychologists. Control participants were interviewed by the first researcher, who was a postgraduate clinical psychology student at the time of data collection. All diagnosticians were extensively trained in the use of the ADIS. Parents and children were interviewed separately and in line with previous research, a combined diagnosis was obtained as per the procedure outlined in Silverman and Albano (1996). Each diagnosis received a Clinical Severity Rating (CSR) from 0 to 8 , where a rating of $0-3$ was considered to be a subclinical presentation and ratings of 4 and over as clinical presentations. Each clinical family in the current study subsequently participated in a research trial within our Centre, thus the inter-rater reliabilities for the main trial will be reported. Inter-rater reliability (kappa) was 0.84 for the presence of a principal anxiety diagnosis and 0.82 for any anxiety diagnosis (Hudson et al. 2013). The ICC for CSR was 0.89.

Participants were recruited to the anxious group on the basis of a composite diagnosis of one or more anxiety disorders with a CSR of four or more on the primary diagnosis. Children with a single diagnosis of Specific Phobia were excluded because it was unlikely that the discrete and distinct nature of their anxiety would impact parental expectations or 
their actual performance during the speech task. Participants were recruited to the anxious/depressed group based on a composite diagnosis of anxiety plus comorbid depression or dysthymia with CSRs of four or more. All anxious/depressed participants had a primary diagnosis of anxiety, with the exception of two participants who had a primary diagnosis of Major Depressive Disorder (MDD) with comorbid anxiety with a CSR of six or higher.

In the anxious group, the distribution of any diagnosis are as follows: $40 \%$ were diagnosed with Separation Anxiety Disorder (SAD), $79 \%$ with Generalized Anxiety Disorder (GAD), $61 \%$ with Social Phobia, $42 \%$ with a Specific Phobia, $7 \%$ with Panic Disorder, and $5 \%$ with Obsessive Compulsive Disorder (OCD); $12 \%$ with Attention Deficit Hyperactivity Disorder (ADHD); $7 \%$ with Oppositional Defiant Disorder (ODD), and $7 \%$ with another diagnoses, which consisted of enuresis, sleep terror, and selective mutism. Within the anxious/depressed group, $60 \%$ were diagnosed with MDD, $40 \%$ with Dysthymia, $80 \%$ with GAD, $80 \%$ with Social Phobia, $40 \%$ with SAD, $33 \%$ with Specific Phobia, $10 \%$ with Panic Disorder, $10 \%$ with OCD, and $10 \%$ with ADHD. In the anxious groups, $91 \%$ had at least one secondary diagnosis. Of the anxious children with a comorbid diagnosis, all had a secondary diagnosis that included anxiety disorders, and some had an additional disorder outside the anxiety spectrum (26\%). Other secondary diagnoses consisted of ADHD (5 children), ODD (three children), selective mutism (1 child), sleep terrors ( 1 child), and enuresis (1 child). In the anxious/depressed group, three children had a secondary diagnosis of ADHD.

\section{Measures}

Parent/Child Speech Preparation Task Using a paradigm developed by Hudson and Rapee (2001), the mother/child dyads undertook a speech task that was designed to be anxietyprovoking for the child, and to elicit parenting behaviors that are putatively involved in the development and maintenance of anxiety (e.g., over-controlling). The mother and child were seated at a table with blank paper and pens. The mother was informed that her child would be required to prepare a short speech to present to a video camera (two, three or four minutes, depending on the child's age). Five minutes was allowed for preparation of the speech. During this time, mothers remained at the table with their child for support. Notes could be used during the speech. Mothers were told, "This task is for your child to do, but you can help them if you feel that they are struggling".

Child Speech At the end of five minutes speech preparation, the researcher re-entered the room and requested the child to prepare to present the speech. The researcher and the parent left the room to watch the recording of the child's speech on a video monitor in an adjoining room. While some children did not speak for the entire time, and some left the camera field, no child actually left the room if they agreed to do the speech. Nine children prepared, but then felt unable to go through with the speech, and were allowed to cease their involvement at this point. Four of these children were from the anxious group, whereas five were from the anxious/depressed group. No children from the control group felt unable to present the speech (also see Data Preparation section).

\section{Child Psychopathology}

Anxiety The Spence Children's Anxiety Scale (SCAS: Spence 1997) was included to provide a measure of child anxiety symptom severity. The SCAS is a child self-report and parentreport measure of anxiety in children and is rated on a 4-point Likert scale. An overall SCAS-C and-P score can be calculated by summing the scores of the items. Both the SCAS-C and -P have adequate psychometric properties with good internal reliability, acceptable test-retest reliability and sound validity (Nauta, et al. 2004; Spence 1998; Spence et al. 2003). Reliabilities of total scores were good in the current sample for child (SCAS-C, $\alpha=0.89$ ) and mother report (SCAS-M, $\alpha=0.95$ ).

Depression The Short Mood and Feelings Questionnaire (SMFQ: Angold, et al. 1995) is a 13-item questionnaire that briefly covers the core set of symptoms related to depression. In our study, both the child self- and parent-report version of the SMFQ was administered. The SMFQ yields a single overall score for depression symptom severity. It discriminates well between depressed and non-depressed samples and has good internal consistency and adequate test-retest reliability (Angold et al. 2002). Reliabilities of total scores were adequate in the current sample for the parent SMFQ $(\alpha=0.87)$ and child SMFQ $(\alpha=0.91)$.

\section{Maternal Psychopathology and Expectancy Ratings}

Pre-Task Questionnaire The Speech Task Parent Rating (STPR) measured maternal expectations of how well their child would perform in the speech task, both in absolute terms and relative to their peers. The ST-PR comprised 16 items of which 14 questions were based directly on previous work by Kortlander et al. (1997) on maternal expectations. The first 15 items measured mother's expectations in absolute terms, and were rated on a Likert scale of $1-5$, where $1=$ Strongly disagree and $5=$ Strongly agree. The final question was a measure of the mother's expectations of child performance relative to their peers (i.e., How well do you think your child will do on this task when compared to other children his/her age?) and was rated from $1=$ Much worse to $5=$ Much better than average (NB. 3 = About the Same). 
Parent Psychopathology The Depression Anxiety Stress Scales (DASS; Lovibond and Lovibond 1995a) were administered to mothers following their completion of the taskrelated questionnaires. This scale assesses maternal stress, depressive, and anxiety symptoms. The DASS consists of 21 items rated on a 4-point Likert scale. The DASS has good validity and discriminates well between depression and anxiety (Lovibond and Lovibond 1995b). Reliabilities of total scores were good in the current sample $(\alpha=0.95)$.

Social Desirability A short-form of the Marlowe-Crowne Social Desirability Scale (M-C Form C; Reynolds 1982) was administered to mothers to provide a measure of how likely it was that they were responding to questions about their child in a socially desirable or self-enhancing manner. The M-C Form C consists of 13 questions on a 5 -point Likert scale $(1=$ Not at all to $5=$ Extremely) and has been shown to be a valid and reliable instrument for the assessment of response bias in relation to social desirability (Reynolds 1982). Reliabilities of total scores were adequate in the current sample $(\alpha=0.77)$.

Objective Rater's Measures To provide an objective rating of speech quality that was directly comparable to the mothers' pre-speech expectation ratings, each child's speech video was rated by a blind coder on the 10 items from the ST-PR pre-task questionnaire that could be directly assessed by an objective observer. The primary coder, a post-graduate student trained in the use of the ST-OR scoring system, coded all videos, and was unaware of the child's diagnostic status. The first researcher coded $25 \%$ of the sample, selected at random, for reliability purposes. For the first nine items that measured the child's performance in absolute terms, a rating was made for each one-minute segment of the speech. The items were: child stays in room, child stays in video view, no excessive pauses, child has appropriate voice, child concentrates on task, eye contact, child shows excessive body movement, child does not become upset/embarrassed, and child has fun, child shows excessive body movement (See Supplementary Material for more information about the coding). The first item of the STOR was removed as it had no variability given that no child who attempted the speech left the room. The item was therefore eliminated from the subsequent factor analysis (see below). As speeches varied in length according to age (e.g., two, three or four minutes), the scores for each minute of the speech were averaged. The final question was a measure of the observer's ratings of child performance relative to their peers (i.e., Overall rating of the child's speech compared to most children his/her age) and was rated from $1=$ Much worse, to $3=$ Average, to $5=$ Much better than average. Inter-rater reliability was calculated using Shrout and Fleiss' (1979) model 2 (Raters random). Analyses showed good inter-rater reliability on the various items ranging from 0.82 to 0.97 .
Procedure

The university's Ethics Review Committee approved the study. Mothers provided written informed consent and children verbal assent to participate in this study. Mothers and children from the control group completed demographics, SCAS, SMFQ and ADIS at time of the speech task. Families from the clinical groups completed demographics, SCAS, and SMFQ and ADIS when they initially presented to the Anxiety Clinic for assessment.

Immediately prior to the speech task, mothers completed the pre-task questionnaire pertaining to the mother's perceptions of their child's speech ability. Mothers remained with their children during the speech preparation task and then watched their child's speech performance via a video monitor. Subsequently, mothers completed the M-C Form C and DASS. Normal controls recruited from the community received financial reimbursement for their participation, while those recruited from the first year psychology pool received course credit.

\section{Data Preparation}

Derivation of Mother and Observer Behavioral Rating Scores Bivariate data for the ST-PR and ST-OR individual items were examined to determine if the items could be fitted into two factors with one accounting for behaviors typical of a good speech, such as making eye contact, and a second factor accounting for behaviors more related to managing anxiety, such as not becoming upset during the speech. Z-scores were used so that data on the mother's scale would be directly comparable to data on the observer's scale. Factor analyses of the maternal and observer data were performed separately using unweighted least squares extraction and oblimin rotation. The eight items included in each analysis were: child stays in video view, no excessive pauses, child has appropriate voice, child concentrates on task, eye contact, child shows excessive body movement, child does not become upset/ embarrassed, and child has fun. The analyses failed to support a two factor solution for either sets of data. Instead the items seemed to load on single factor, resulting in a single maternal and observer report scale derived from the sum of the z-scores. One item was removed (child shows excessive body movement) from both the maternal and objective rater scales because it failed to contribute to the overall Cronbach's alphas. The resulting Cronbach's alphas were 0.90 and 0.83 respectively for mothers' and observer's ratings of children's speech behaviors. Together, a total of two dependent variables were available for analysis per rater (mother and objective observer): an absolute rating of the child's performance (Absolute Speech Rating) and a relative rating for the child's performance in comparison to peers (Relative Speech Rating). For ease of terminology, observer's ratings will be preceded by the 
word observed (i.e., observed Absolute Speech Rating and observed Relative Speech Rating) and maternal ratings will be preceded by the word expected (i.e., expected Absolute Speech Rating and expected Relative Speech Rating).

Imputation of Missing Data As nine children refused to complete the speech task, all of which were from one of the clinical groups, the observer's data contained data that was not missing completely at random (over all groups). Indeed, primary diagnosis CSR was different between children who completed the task and children who refused the task $(p<0.01)$. That is, children who refused exhibited significantly higher primary diagnosis CSR $(M=7.22, S D=0.67)$ than children who complied $(M=4.06, S D=3.03)$. Ignoring this missing data would have biased the data for the clinical groups, by eliminating those cases where speech performance was at its poorest, i.e. no speech was completed due to anxiety about the task. We used Jaccard and Guilamo-Ramos' (2002) recommendation of the expectation-maximisation method to overcome systematic bias by imputing missing data in repeated measures designs. Both mothers' and the observer's ratings were converted to z-scores, and then an expectation maximisation procedure was implemented to impute the missing speech data using speech task data and level of child anxiety reported on the SCAS of the complete cases. All reported analyses were completed including the imputed data ${ }^{1}$.

\section{Results}

\section{Preliminary Findings}

Table 1 provides descriptive data for child and maternal psychopathology. As expected, one-way Analyses of Variance (ANOVAs) revealed significant differences between the three groups on child self-reported anxiety, $F(2,114)=33.40, p<0.001$, mother-reported child anxiety, $F(2,114)=72.67, p<0.001$, child self-reported depression, $F(2,109)=23.64, p<0.001$, and motherreported child depression, $F(2,109)=70.24, p<0.001$. As can be seen in Table 1, children in the clinical groups displayed higher mother- and child-rated anxiety scores than children in the control group, whereas children in the anxious/depressed group displayed higher child-rated and mother-rated depression scores than children in the control and anxious group. Children in the anxious group also displayed higher motherrated depression scores than children from the control group. ANOVAs for maternal psychopathology were significant for stress, $F(2,113)=5.06, p=0.008$, anxiety, $F(2,113)=8.93$, $p<0.001$, and depression, $F(2,113)=3.34, p=0.039$. Follow-

\footnotetext{
$\overline{{ }^{1} \text { Imputed data }}$ and non-imputed produced similar results for all overall tests and contrasts.
}

up contrasts revealed that mothers of non-clinical children reported significantly less stress, $F(1,115)=10.11, p<0.05$, anxiety, $F(1,115)=13.91, p<0.01$, and depression, $F(1,115)=6.60$, $p<0.05$, than the children in the clinical groups. However, there were no differences between mothers in the anxious and anxious/depressed group on maternal stress, anxiety or depression ( $p$ range: $0.12-0.78$ ). Finally, no significant differences in mothers' social desirability scores were found between the control, anxious, and anxious/depressed groups (respectively $M=2.66, \quad S D=0.50, \quad M=2.70, \quad S D=0.52, \quad$ and $M=2.67$, $S D=0.55, p>0.05)$.

Associations Between Dependent and Independent Variables

Correlations between the four dependent variables (Speech task behaviors) and the independent variables (Maternal and Child psychopathology) are provided in Table 2. Associations were found between the dependent variables and maternal anxiety, child self-reported anxiety and depression, and mother-reported child anxiety and depression. These associations were generally weak to moderate, and in the expected directions. The dependent variables were not associated with child age, sex, mothers' socially desirable responding or maternal stress or depression. Among the clinical children only, results showed that the number of co-morbid anxiety disorders was associated with observers' Relative Speech Ratings, and with mothers' expectations on Relative Speech Ratings.

\section{Maternal and Observer Ratings of Child Speech}

Table 3 provides the means and standard deviations for the Absolute Speech Rating and Relative Speech Rating performance by mothers and the observer. As can be seen from Table 3 mothers in the control group rated their own child's expected performance as better than their peers (a rating of 3 indicated about the same), whereas the objective observer rated control children's actual performance as average (i.e., a rating of 3) overall. Mothers of clinical group children expected their child's performance to be below average compared to peers, in line with how the observer rated their actual performance.

\section{Reporter Bias: Overall Group and Rater Effects}

To examine potential reporter bias, a repeated measures Multivariate Analysis of Variance (MANOVA) was carried out with the Group variable (control, anxious, anxious/depressed) as between-subjects factor, Rater (mother or observer) as within-subjects measure and the Absolute Speech Rating (ratings of the child's speech behaviors) and Relative Speech Rating (rating of the child relative to peers) as the two dependent measures. Maternal anxiety and depression were included as covariates in the initial analyses, but failed to contribute 
Table 1 Descriptive statistics for child and parent psychopathology by group

$D A S S$ depression anxiety and stress scales, SCAS Spence Children's Anxiety Scale, $S M F Q$ short mood and feelings questionnaire

Row means with differing subscripts differ significantly at $p<0.05$ on Bonferroni-Holm corrected t-tests

\# Trend $(p<0.10)$ between Controls and Clinical groups after Bonferroni-Holm correction

\begin{tabular}{|c|c|c|c|c|c|c|c|c|c|}
\hline & \multicolumn{3}{|c|}{ Controls } & \multicolumn{3}{|c|}{ Anxious } & \multicolumn{3}{|c|}{ Anxious/Depressed } \\
\hline & $\mathrm{n}$ & M & SD & $\mathrm{n}$ & M & SD & $\mathrm{n}$ & M & SD \\
\hline \multicolumn{10}{|l|}{ Child psychopathology } \\
\hline SCAS - child report & 44 & $15.36_{\mathrm{a}}$ & 10.07 & 43 & $31.191_{b}$ & 15.22 & 30 & $40.53_{\mathrm{c}}$ & 15.53 \\
\hline SCAS - maternal report & 44 & $7.59_{\mathrm{a}}$ & 5.26 & 43 & $36.37_{\mathrm{b}}$ & 15.57 & 30 & $37.03_{\mathrm{b}}$ & 15.32 \\
\hline SMFQ - child report & 44 & $2.75_{\mathrm{a}}$ & 3.52 & 40 & $4.75_{\mathrm{a}}$ & 4.41 & 28 & $10.68_{\mathrm{b}}$ & 6.83 \\
\hline SMFQ - maternal report & 44 & $.98_{\mathrm{a}}$ & 1.30 & 39 & $5.64_{b}$ & 3.83 & 27 & $10.54_{c}$ & 4.62 \\
\hline \multicolumn{10}{|l|}{ Maternal psychopathology } \\
\hline DASS - stress & 44 & $8.73_{\mathrm{a}}$ & 6.94 & 43 & $14.05_{\mathrm{b}}$ & 10.16 & 29 & $13.45_{\mathrm{b}}$ & 7.40 \\
\hline DASS - anxiety & 44 & $1.50_{\mathrm{a}}$ & 2.40 & 43 & $6.28_{\mathrm{b}}$ & 7.23 & 29 & $3.86_{\mathrm{ab}}$ & 4.98 \\
\hline DASS - depression & 44 & $3.28^{\#}$ & 4.79 & 43 & 5.96 & 6.61 & 29 & 6.48 & 6.34 \\
\hline
\end{tabular}

significantly to the explanation of the dependent variables and were therefore removed from subsequent analyses.

Results of this analysis showed a significant betweensubjects effect of Group, with the overall Wilks' $F(4,226)$ for Group $=16.08, p<0.001, \mathrm{y}_{\mathrm{p}}{ }^{2}=0.22$. Furthermore, for the within subjects variables, a significant effect of Rater, $F(2,113)=11.04$, $p<0.001, \mathrm{y}_{\mathrm{p}}{ }^{2}=0.17$, and a significant Rater $\mathrm{x}$ Group interaction was found, Wilks' $F(4,226)=4.56, p=0.002, \mathrm{y}_{\mathrm{p}}{ }^{2}=0.07 .^{2}$ The Rater $x$ Group interaction represented differences in discrepancies between raters for each group, or a reporting bias. Followup contrasts were used to explore these effects and the significant interaction, directly comparing the level of reporter bias in each group in comparison to the other groups, separately for each of the two dependent variables.

\section{Follow-Up Pairwise Comparisons: Rater and Group Effects}

To control for Type I error over multiple contrasts, alpha was maintained at 0.008 (an overall alpha $=0.05 / 6$ ) by using Bonferroni-Holm corrections. Multiple pairwise comparisons examined the discrepancies between raters for each group for the two dependent variables individually. For the dependent variable Absolute Speech Rating, there were significant differences between the maternal and observer ratings in the control group only, $t(114)=3.65, p<0.001$, Cohen's $d=1.04$. Mothers of controls expected their children to perform significantly better than how they actually performed as rated by the objective observer. For the Relative Speech Rating, there also was a significant difference between raters in the control

\footnotetext{
${ }^{2}$ A notable possibility that was not explicitly explored in the main analysis is that only anxious mothers exhibit reduced expectancy of an anxious child (Cobham et al. 1999). That is, maternal anxiety may interact with child anxiety and/or rater to produce lower expectations of the child's performance. We explored these interactions in a supplementary mixed model analysis given that maternal anxiety was measured using a continuous variable (DASS anxiety subscale). We did not observe any significant interactions (2- or 3-way) for Absolute Speech Ratings or Relative Speech Ratings ( $p s<0.05)$.
}

group only, $t(114)=4.02, p<0.001, d=0.95$, with control group mothers expecting their child to perform above average, whereas the observer rated their actual performance as average. Together, these results indicate that there were large effects for Rater upon Absolute Speech Rating and Relative Speech Rating in the control group. These effects were not present in either of the clinical groups.

Furthermore, multiple pair-wise comparisons were used to examine the between-group differences for each rater on the Absolute Speech Rating and Relative Speech Rating. Results showed that children in the control group scored significantly better than children in either the anxious group, $t(85)=3.09$, $p=0.002, d=0.67$, or the anxious/depressed group, $t(72)=3.39$, $p=0.001, d=0.80$ on observed Absolute Speech Rating. There were no significant differences in observed Absolute Speech Ratings between anxious and anxious/depressed children. On the other hand, mothers of control children expected their child to perform better in term of their expected Absolute Speech Rating than did either mothers of anxious children, $t(85)=7.95$, $p<0.001, d=1.73$ or mothers of anxious/depressed children, $t(72)=8.46, p<0.001, d=1.99$. Children in the anxious and anxious/depressed groups did not differ significantly on expected Absolute Speech Rating. Overall, effect sizes indicated large differences in Absolute Speech Ratings between the control and both clinical groups.

When looking at the Relative Speech Rating, the objective rater scored the children from the control group overall as average compared to their peers. However, this was still significantly better than their anxious, $t(85)=3.57, p=0.001, d=0.77$, and marginally better than anxious/depressed counterparts, $t(72)=2.70, p=0.008, d=0.64$, who were considered by objective raters as below average. The two clinical groups did not differ significantly on observed Relative Speech Ratings. Mothers of non-clinical children expected that their child's performance would be better than that of their peers, rating them above average overall. Their expected Relative Speech Ratings were significantly higher than the expected Relative Speech Ratings for anxious children, $t(85)=5.83, p<0.001$, 


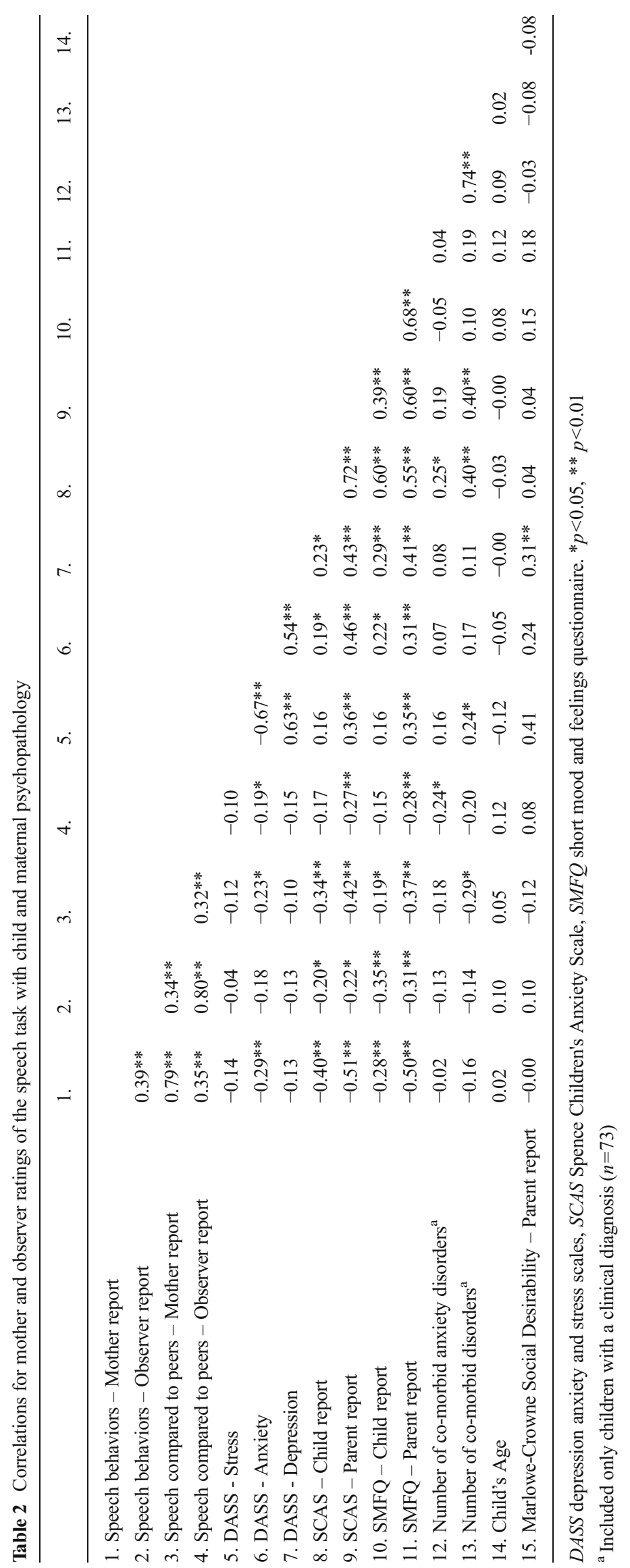


Table 3 Means and standard deviations for mothers' and observers' absolute speech ratings and relative speech ratings across the three groups

\begin{tabular}{|c|c|c|c|c|c|c|}
\hline & \multicolumn{2}{|c|}{ Controls $(n=44)$} & \multicolumn{2}{|c|}{ Anxious $(n=43)$} & \multicolumn{2}{|c|}{ Anxious/Depressed $(n=30)$} \\
\hline & M & SD & M & SD & M & SD \\
\hline \multicolumn{7}{|l|}{ Absolute speech ratings } \\
\hline Observer report - aggregated z-scores & $* 1.75_{\mathrm{a}}$ & 2.92 & $-1.29 \mathrm{~b}$ & 4.25 & $-1.92_{\mathrm{b}}$ & 6.61 \\
\hline Maternal report - aggregated z-scores & $* 4.72_{\mathrm{a}}$ & 2.77 & $-2.33_{\mathrm{b}}$ & 4.67 & $-3.57_{\mathrm{b}}$ & 4.93 \\
\hline \multicolumn{7}{|l|}{ Relative speech ratings } \\
\hline Observer report Likert score & $* 3.07_{\mathrm{a}}$ & 0.55 & $2.46_{\mathrm{b}}$ & 0.84 & $2.57_{\mathrm{b}}$ & 0.99 \\
\hline Maternal report Likert score & $* 3.66_{\mathrm{a}}$ & 0.68 & $2.74_{b}$ & 0.69 & $2.67 \mathrm{~b}$ & 0.84 \\
\hline
\end{tabular}

Row means with differing subscripts differ significantly at $p<0.05$ on Bonferroni-Holm corrected t-tests

* Mothers of controls expected their children to perform significantly better than their children were rated by an objective observer for both relative to peers ratings and aggregated z-scores for observable speech behaviors $(p<0.05)$

$d=1.26$, and anxious/depressed children, $t(72)=5.73, p<0.001$, $d=1.35$. The mothers of the anxious and anxious/depressed children did not differ significantly in terms of how they expected their child to perform relative to an average child. Effects sizes for observer report were medium in contrast to the large effect sizes for differences between control and clinical groups on maternal Relative Speech Ratings.

\section{Follow-Up Contrasts on Rater x Group Interactions}

For the Absolute Speech Rating, the interaction contrasts showed that the groups differed significantly on the discrepancies between the raters. The contrasts measuring the discrepancies between maternal and observer ratings were significant for controls versus the anxious group, $t(85)=3.48$, $p=0.001, d=0.65$, and controls versus the anxious/depressed group, $t(72)=3.62, p<0.001, d=0.68$. Similarly, the contrast for the differences in rater discrepancy between the control and the combined clinical groups was also significant, $t(114)=4.17, p<0.001, d=0.78$. As indicated by the scores in Table 3, this means that mothers in the control group expected their child to perform significantly better than how the objective observer rated their child's performance, suggesting a positive bias. This positive bias was not present in either the anxious or anxious/depressed group. The two clinical groups did not differ significantly in their levels of reporter bias on the Absolute Speech Rating dependent variable. None of the interaction contrasts for the Relative Speech Rating were significant.

\section{Anxiety Types and Rating Effects}

We examined whether speech ratings were poorer for certain types of primary anxiety diagnoses. We elected to examine primary diagnoses of GAD $(n=29)$, SAD $(n=12)$ and Social Phobia $(n=21)$ as they were the most common primary diagnoses. We reclassified Group into Anxiety Type. For instance, GAD Anxiety Type was GAD vs. all other primary diagnosis. MANOVAs were conducted for each of the selected anxiety disorders. Table 4 provides the means and standard deviations for ratings of Absolute Speech Ratings and Relative Speech Ratings by mothers and observer across the three anxiety disorders for primary diagnosis. Results revealed that children with a primary diagnosis of Social Phobia received lower Absolute Speech Ratings, regardless of Rater, than children with other diagnosis, $F(1,71)=11.04, p<0.001, \mathrm{y}_{\mathrm{p}}{ }^{2}=0.06$. There were no other main effects or interactions for Social Phobia or any of the other disorders (all $p \mathrm{~s}<0.05$ ).
Table 4 Means and standard deviations for mothers' and observers' absolute speech ratings and relative speech ratings for children with a primary diagnosis of GAD, SAD, and social phobia

\begin{tabular}{|c|c|c|c|c|c|c|}
\hline & \multicolumn{2}{|c|}{ GAD $(n=29)$} & \multicolumn{2}{|c|}{$\operatorname{SAD}(n=12)$} & \multicolumn{2}{|c|}{ Social phobia $(n=21)$} \\
\hline & M & SD & M & $\mathrm{SD}$ & M & $\mathrm{SD}$ \\
\hline \multicolumn{7}{|l|}{ Absolute speech ratings } \\
\hline Observer report - aggregated z-scores & -1.50 & 5.28 & .01 & 4.57 & -2.68 & 6.27 \\
\hline Maternal report - aggregated z-scores & -2.40 & 4.35 & -1.55 & 4.69 & -4.70 & 4.75 \\
\hline \multicolumn{7}{|l|}{ Relative speech ratings } \\
\hline Observer report Likert score & 2.60 & 0.81 & 2.55 & 1.08 & 2.23 & 0.86 \\
\hline Maternal report Likert score & 2.76 & 0.68 & 2.83 & 0.71 & 2.52 & 0.81 \\
\hline
\end{tabular}




\section{Discussion}

The current study examined whether mothers of anxiety disordered children exhibited a positive bias towards their child, like mothers of non-anxious children, when assessing their child's ability in a stressful performance situation. Maternal expectancy ratings were compared to objective observer ratings to determine whether patterns in maternal expectations reflected accurate assessments of the child's skill and coping ability. Our hypothesis that mothers of anxious children might lack a positive bias compared to mothers of non-clinical children was confirmed. Most of the results were in line with our predictions. That is, our data supported the hypothesized positive bias for controls in relation to mothers' expectations about their child's performance, both when assessed as absolute ratings and when mothers were asked about their child's performance relative to their peers (i.e. compared to other children their age). Specifically, mothers of non-clinical children expected their children to be more capable and to perform better compared to their peers than that of their actual performance as rated by the objective rater. In line with our predictions, mothers of anxious and anxious/depressed children did not demonstrate the same positive bias that was observed in mothers of non-clinical children. The mothers of clinical children expected their child to perform worse than the average child, which was commensurate with the objective observer's ratings.

An important issue to address when assessing maternal bias is to consider the credibility of each of the reporters. In this case, the observer rated the control group as average overall, when compared to other children. There is some indirect evidence that the observer's overall rating appears credible. For instance, the observer rated the children in the two clinical groups as significantly less skilled in comparison to an average child, which is consistent with previous research that have shown that that both anxious and depressed children may demonstrate poorer social skills in some instances (Kennedy et al. 1989; Strauss et al. 1989). Moreover, there was good to excellent agreement between two independent objective observers, further suggesting that the observer ratings were relatively accurate.

The results of the current study confirmed the hypothesis that mothers of children with internalizing disorders do not show a positive self-serving bias when anticipating their child's performance. However, no evidence was found for a negative bias (i.e., being overly pessimistic) either. As there were no significant differences between mothers and objective raters in either of the clinical groups, this suggests that mothers of anxious and anxious/depressed children were realistic in their parental expectations. They did expect their children to perform worse than their peers, and predicted lower performances on the absolute speech ratings, but these were consistent with the objective observer's ratings. These findings dispute the viewpoint that parents of children with anxiety or mood problems are overly pessimistic or negatively biased in their expectations of their children (Cobham, et al. 1999; Kendall and Chansky 1991; Kortlander, et al. 1997). In contrast, the data suggests that mothers of internalizing children simply do not demonstrate the pervasive positive, selfserving bias seen in parents of non-clinical children. This is consistent with Gretarsson and Gelfand's (1988) findings, which showed that mothers may be less motivated to maintain positive perceptions of their child when the child is more difficult to handle. Although our current study and previous findings cannot answer the question as to why mothers of difficult children, or children with clinical anxiety and depressive disorders display a lack of a positive bias, it is possible that this lack of a positive bias serves as a alleviation of the parental sense of responsibility for the child's difficulties and to maintain the parent's self-esteem. Future studies are necessary to investigate these explanations.

Among the clinical groups, Absolute Speech Ratings across raters were significantly lower for children with a primary diagnosis of Social Phobia compared to other primary diagnoses. This was not surprising given that speech tasks are typically one of the most challenging tasks for individuals with Social Phobia. However, an unexpected finding in the clinical groups was the absence of maternal negative bias in the anxious/depressed group. Based on previous findings (Hughes and Gullone 2008; McLeod, et al. 2007), we expected that maternal negativity or criticism might have been more of a characteristic of the anxious/depressed group rather than the anxious group, which was not found in the current study. It is possible that when maternal negativity was observed in previous studies, it was a response to the child's behaviors or negative affect (Udy and Hudson 2008). Our study differed from previous studies in that mothers were asked for their ratings prior to the experimental task, rather than after the child's speech preparation, which for many clinical dyads involved conflicted parent-child interactions. Indeed, it may be that parent-child interactions during speech preparation led to differences in the children's performance when rated by the observer. From previous research, we know that parents interact differently with an anxious child compared to a nonanxious child during speech preparation tasks. Thus, it is possible that differing interactions between parent and child during the preparation could lead to different performances.

An aforementioned point of difference between the mother and observer ratings should be emphasised here: maternal ratings were based on their expectations of their child before the speech task while observer ratings were based on actual speech performance. This is a subtle, yet important, difference that limits the extent to which we can directly compare maternal and observer ratings. Thus we do not know, for example, whether mothers may have adjusted their ratings after watching the speech, and whether this occurred differentially 
for mothers of anxious and non-clinical children. Future studies may consider also tracking maternal ratings of their child's performance after the speech task and directly compare this to observer ratings. This may reveal important factors (e.g., maternal psychopathology) that can lead to re-appraisal of their child's abilities following observation of their child's performance.

This study also replicated previous findings that indicate anxiety is more prevalent in mothers of anxious children than mothers of non-anxious controls (e.g., Last et al. 1991). We also showed that maternal anxiety shared a positive association with reduced maternal expectations on ratings. However, neither maternal depression nor anxiety contributed significantly to maternal ratings in our analyses comparing maternal ratings to observer ratings. That is, there was little indication that maternal anxiety or depression was a source of reporter bias, over and above differences due to the child's diagnosis. This is surprising given that levels of parental psychopathology are often related to discrepancies compared to other informants (Des Los Reyes and Kazdin 2005). We also explored whether maternal psychopathology interacted with child anxiety status and rater to produce lower Absolute and Relative Speech Ratings (see Footnote 1). However, we did not find any interacting effects with maternal psychopathology, further supporting the viewpoint that maternal psychopathology did not play a role in our current findings. It is worth noting that we may have been under-powered to detect interaction effects, especially a three-way effect of maternal psychopathology, child psychopathology, and rater. Furthermore, in this study maternal psychopathology was assessed by selfreport measures, and mean depression and anxiety scores for mothers of anxious and anxious/depressed children were in the non-clinical range. In future studies, parental anxiety and mood disorders may need to be assessed by gold-standard diagnostic methods as a basis for diagnosis rather than the self-reported measures used in the current study. It also remains to be seen whether mothers with clinical levels of anxiety or depression might also exhibit more pessimistic expectations of their children with internalizing difficulties when compared to an objective observer's ratings. This may further elucidate the influence of parental psychopathology on expectancy bias.

It is important to consider alternative explanations for the data and further limitations of this study. This study asked parents to rate their children on a task that was likely to be anxiety-provoking, and as such the ratings may have held special significance for parents of anxious children. Mothers of anxious children may be more sensitized to their child's issues around performing the experimental task and this might have increased their expectancy accuracy relative to controls. Moreover, due to the nature of the experimental task, the findings of this study may not generalize to other domains of the child's life. Specifically, the lack of parental positive bias seen in the clinical groups may only be evident in situations where the mother is particularly sensitized to the child's difficulties (i.e., those situations that are anxiety-provoking for their child). However, it is also possible that if mothers from the clinical groups were asked to predict their child's academic performance or sporting prowess, that they may also demonstrate a positive bias, similar to the control mothers. Further research should consider including maternal predictions and expectations across other child domains in order to rule out this possibility. Replications showing parental positive bias in domains such as child intelligence and developmental level with anxious children as well as community controls would be informative.

There are two other limitations worth noting about our results. First, we did not detect age effects on ratings, which is unusual given that one would expect older children to be given higher ratings on Absolute Speech Ratings than younger children. Older children will have been exposed to more situations for public speaking (e.g., presenting at school) and therefore, should be more experienced in delivering speeches. We suspect that both the mother and observer automatically adjusted their ratings relative age on the Absolute Speech Ratings, thus accounting for the lack of age effects.

Our results may also have significant contributions to the theoretical and clinical understanding of childhood anxiety disorders. For instance, the lack of a positive expectancy bias displayed by mothers of clinical children may play an important role in the development and maintenance of childhood anxiety disorders. Previously, several researchers have proposed that parents of anxious children may be more attuned to threat and that this threat-bias can be seen in the parents' evaluations of their child's abilities too (Bar-Haim, et al. 2007; Creswell, et al. 2005). These reduced parental expectations of their child's coping abilities may be one possible mechanism by which anxiety may be transmitted from parent to offspring (Rapee 1997). Specifically, parents with greater threat-bias may increase deleterious parental styles such as over-involvement and excessive control, or verbally convey to the child the costs of making mistakes. Such parenting behaviours may be utilised in an attempt to minimise threat but in fact, undermine the child's confidence and increase anxiety. It is important to note that recent research suggests that it is not the case that anxious parents, who should have greater threat bias, exhibit more over-controlling and anxious behaviours whilst helping their child (non-anxious) prepare for a speech task (Becker and Ginsburg 2011). That study differs from our study in that they recruited non-anxious children but targeted differences in maternal anxiety (Becker and Ginsburg 2011). The anxious mothers in that study did not report lower expectations about their non-anxious child's performance compared to non-anxious mothers. Our current study, along with Becker and Ginsburg's (2011) findings, suggest that lower positive bias is mainly due to the anxiety status of the child and not the 
parent. It remains to be seen when the absence of positive bias about an anxious child, and not parental anxiety per se, is the critical ingredient that predicts greater parental over-control and anxiety during the speech preparation task. An important limitation in the current study is that we did not record and code parent-child interactions during the speech preparation, which may have provided a more nuanced interpretation of how the lack of positive bias may have been associated with overt parental behaviours.

Our study may also be relevant to the literature on informant discrepancy and its application to childhood psychopathology. Specifically, recent research has stressed the importance and meaning of informant discrepancy beyond measurement error when evaluating a child or adolescent's ongoing difficulties (Des Los Reyes 2011; Des Los Reyes and Kazdin 2005; Laird and Weems 2011; Weems et al. 2011). For example, the lack of agreement on the presence of a childhood anxiety problem between a parent and child has been shown to predict poorer Cognitive Behavioral Therapy outcomes (Panichelli-Mindel et al. 2005). In our study, an agreement between observer and parent was found within a clinical sample but not in a non-clinical sample. This observation could be extended in future studies to evaluate whether the presence or absence of this positive bias in parents (i.e., agreement or disagreement with an independent observer; see also Laird and Weems 2011 for appropriate methods of evaluating informant discrepancies) of internalizing children might be a sensitive measure for treatment outcome, and whether the lack of positive bias in parents should be a target for intervention in future treatment frameworks. It is also worth noting that any aim of increasing positive bias in parents of anxious children is at odds with the fundamental principles of cognitive behavioural therapy, which aims at increasing realistic thinking among anxious children and his or her parent. However, in this instance, it is possible that increasing some unrealistically optimistic biases in parents of anxious children can critically convey confidence to the anxious child about their abilities, and may have a positive impact on the child's performance in stressful situations. Of course, experimental studies are needed to verify whether parental positive bias plays a causal role in improving the anxious child's confidence, as well as their actual performance in a stressful task. Such studies will be especially informative for new and more effective, family-based treatment frameworks for childhood anxiety disorders.

Open Access This article is distributed under the terms of the Creative Commons Attribution License which permits any use, distribution, and reproduction in any medium, provided the original author(s) and the source are credited.

\section{References}

Ackermann, R., \& DeRubeis, R. J. (1991). Is depressive realism real? Clinical Psychology Review, 11, 565-584.

Angold, A., Costello, E. J., Messer, S. C., Pickles, A., Winder, F., \& Silver, D. (1995). The development of a short questionnaire for use in epidemiological studies of depression in children and adolescents. International Journal of Methods in Psychiatric Research, 5, 1-12.

Angold, A., Costello, E. J., \& Erkanli, A. (1999). Comorbidity. Journal of Child Psychology and Psychiatry, and Allied Disciplines, 40, 57-87.

Angold, A., Erkanli, A., Silberg, J., Eaves, L., \& Costello, E. J. (2002). Depression scale scores in 8-17-year-olds: effects of age and gender. Journal of Child Psychology and Psychiatry, 43, 1052-1063.

Bar-Haim, Y., Lamy, D., Pergamin, L., Bakermans-Kranenburg, M. J., \& van IJzendoorn, M. (2007). Threat-related attentional bias in anxious and nonanxious individuals: a meta-analytic study. Psychological Bulletin, 133, 1-24.

Becker, K. D., \& Ginsburg, G. S. (2011). Maternal anxiety, behaviors, and expectations during a behavioral task: relation to children's selfevaluations. Child Psychiatry and Human Development, 42, 320333.

Cobham, V. E., Dadds, M. R., \& Spence, S. H. (1999). Anxious children and their parents: what do they expect? Journal of Clinical Child Psychology, 28, 220-231.

Comer, J. S., \& Kendall, P. C. (2004). A symptom-level examination of parent-child agreement in the diagnosis of anxious youths. Journal of the American Academy of Child and Adolescent Psychiatry, 43, 878-886.

Creswell, C., Schniering, C. A., \& Rapee, R. M. (2005). Threat interpretation in anxious children and their mothers: comparison with nonclinical children and the effects of treatment. Behaviour Research and Therapy, 43, 1375-1381.

Des Los Reyes, A. (2011). Introduction to the special section: more than measurement error: discovering meaning behind informant discrepancies in clinical assessments of children and adolescents. Journal of Clinical Child \& Adolescent Psychology, 40, 1-9.

Des Los Reyes, A., \& Kazdin, A. E. (2005). Informant discrepancies in the assessment of childhood psychopathology: a critical review, theoretical framework, and recommendations for further study. Psychological Bulletin, 131, 483-509.

Gretarsson, S., \& Gelfand, D. M. (1988). Mothers' attributions regarding their children's social behavior and personality characteristics. Developmental Psychology, 24, 264-269.

Hudson, J. L., \& Rapee, R. M. (2001). Parent-child interactions and anxiety disorders: an observational study. Behaviour Research and Therapy, 39, 1411-1427.

Hudson, J.L., Newall, C., Rapee, R.M., Lyneham, H., Schniering, C., Wuthrich, V., et al. (2013). The impact of brief parental anxiety management on childhood anxiety treatment outcomes: a controlled trial. Journal of Clinical Child and Adolescent Psychology. doi:10. 1080/15374416.2013.807734

Hughes, E. K., \& Gullone, E. (2008). Internalizing symptoms and disorders in families of adolescents: a review of family systems literature. Clinical Psychology Review, 28, 92-117.

Jaccard, J., \& Guilamo-Ramos, V. (2002). Analysis of variance frameworks in clinical child and adolescent psychology: advanced issues and recommendations. Journal of Clinical Child Psychology, 31, 278-294.

Kendall, P. C., \& Chansky, T. E. (1991). Considering cognition in anxiety-disordered children. Journal of Anxiety Disorders, 5, 167185.

Kennedy, E., Spence, S. H., \& Hensley, R. (1989). An examination of the relationship between childhood depression and social competence amongst primary school children. Journal of Child Psychology and Psychiatry and Allied Disciplines, 30, 561-573. 
Kessler, R. C., Berglund, P., Demler, O., Jin, R., Koretz, D., Merikangas, K. R., Rush, A. J., Walters, E. E., \& Wang, P. S. (2003). The epidemiology of major depressive disorder: results from the National Comorbidity Survey Replication (NCS-R). Journal of the American Medical Association, 289, 3095-3105.

Kortlander, E., Kendall, P. C., \& Panichelli-Mindel, S. M. (1997). Maternal expectations and attributions about coping in anxious children. Journal of Anxiety Disorders, 11, 297-315.

Laird, R. D., \& Weems, C. F. (2011). The equivalence of regression models using difference scores and models using separate scores for each informant: Implications for the study of informant discrepancies. Psychological Assessment, 23, 388-397.

Last, C. G., Hersen, M., Kazdin, A. E., Orvaschel, H., \& Perrin, S. (1991). Anxiety disorders in children and their families. Archives of General Psychiatry, 23, 185-190.

Lovibond, P. F., \& Lovibond, S. H. (1995a). The structure of negative emotional states: comparison of the Depression Anxiety Stress Scales (DASS) with the Beck Depression and Anxiety Inventories. Behaviour Research and Therapy, 33, 335-343.

Lovibond, S. H., \& Lovibond, P. F. (1995b). Manual for the depression anxiety stress scales. Sydney: Psychology Foundation.

Malhi, P., Kashyap, S., \& Dua, S. (2005). Maternal estimates of mental age in developmental assessment. Indian Journal of Pediatrics, 72, 931-934.

Mathew, A. R., Pettit, J. W., Lewinsohn, P. M., Seeley, J. R., \& Roberts, R. E. (2011). Co-morbidity between major depressive disorder and anxiety disorders: shared etiology or direct causation? Psychological Medicine, 41, 2023-2034.

McLeod, B. D., Weisz, J. R., \& Wood, J. J. (2007). Examining the association between parenting and childhood depression: a metaanalysis. Clinical Psychology Review, 27, 986-1003.

Miller, S. A. (1986). Parents' beliefs about their children's cognitive abilities. Developmental Psychology, 22, 276-284.

Miller, S. A., Manhal, M., \& Mee, L. L. (1991). Parental beliefs, parental accuracy and children's cognitive performance: a search for causal relations. Developmental Psychology, 27, 267-276.

Nauta, M. H., Scholing, A., Rapee, R. M., Abbott, M., Spence, S. H., \& Waters, E. (2004). A parent report measure of children's anxiety: psychometric properties and comparison with child-report in clinic and normal sample. Behaviour Research and Therapy, 42, 813839.

Panichelli-Mindel, S. M., Flannery-Schroeder, E., Kendall, P. C., \& Angelosante, A. G. (2005). Disclosure of distress among anxiety- disordered youth: differences in treatment outcome. Journal of Anxiety Disorders, 19, 403-422.

Rapee, R. M. (1997). Potential role of childrearing practices in the development of anxiety and depression. Clinical Psychology Review, 17, 47-67.

Reynolds, W. M. (1982). Development of reliable and valid short forms of the Marlowe-Crowne Social Desirability Scale. Journal of Clinical Psychology, 38, 119-125.

Shrout, P. E., \& Fleiss, J. L. (1979). Intraclass correlations: uses in assessing rater reliability. Psychological Bulletin, 8, 420-428.

Silverman, W. K., \& Albano, A. M. (1996). Anxiety disorders interview schedule for DSM-IV: Child and parent versions. Boulder: Graywind Publications Incorporated.

Silverman, W. K., Saavedra, L. M., \& Pina, A. A. (2001). Test-retest reliability of anxiety symptoms and diagnoses with anxiety disorders interview schedule for DSM-IV: child and parent versions. Journal of the American Academy of Child and Adolescent Psychiatry, 40, 937-944.

Spence, S. H. (1997). The structure of anxiety symptoms among children: a confirmatory factor analytic study. Journal of Abnormal Psychology, 106, 280-297.

Spence, S. H. (1998). A measure of anxiety symptoms among children. Behaviour Research and Therapy, 36, 545-566.

Spence, S. H., Barrett, P. M., \& Turner, C. M. (2003). Psychometric properties of the Spence Children's Anxiety Scale with young adolescents. Journal of Anxiety Disorders, 17, 605-625.

Strauss, C. C., Lease, C. A., Kazdin, A. E., Dulcan, M. K., \& Last, C. G. (1989). Multimethod assessment of the social competence of children with anxiety disorders. Journal of Clinical Child Psychology, $18,184-189$.

Taylor, S. E., \& Brown, J. D. (1988). Illusion and well-being: a social psychological perspective on mental health. Psychological Bulletin, 103, 193-210.

Taylor, S. E., \& Brown, J. D. (1994). Positive illusions and well-being revisited: separating fact from fiction. Psychological Bulletin, 116, 21-27.

Udy, C.M., \& Hudson, J.L. (2008). Maternal involvement and negativity in anxious and anxious/depressed children: The mediating roles of child negative affect and matter affect and cognitions. Unpublished manuscript.

Weems, C. F., Feaster, D. J., Horigian, V. E., \& Robbins, M. S. (2011). Parent and child agreement on anxiety disorder symptoms using the DISC predictive scales. Assessment, 18, 213-216. 